height and plate thickness; in other words, it should be possible in principle to determine plate thickness indirectly from volcano height. In practice, this will only be possible for active or recently extinct volcanoes because older volcanoes will have been eroded and their heights lost for ever. Moreover, there are almost certainly bound to be some volcanoes which cease activity because of insufficient magma and others that have not yet reached their full height, and in these cases heights will also be too low to conform with any volcano height-plate thickness relationship.

But what do the data show? From the mathematics of the isostatic system, Vogt concludes that, when corrected for the buoyancy of their submerged bases, the height of young oceanic volcanoes should vary as the square root of crustal age. A plot of the relevant observations confirms this relationship 'with a surprisingly low scatter of points about the straight line. Because the data obey the expected relationship, they implicitly support the assumptions upon which the relationship was derived. In other words, the results suggest that most volcano heights are indeed limited by isostasy rather than magma shortage. An increase in volcano height thus implies an increase in plate thickness, and a linear relationship between volcano height and the square root of age implies a similar relationship between plate thickness and square root of age. Hence there is support here for the Parker-Oldenburg model.

On the other hand, this does not mean that the constant thickness hypothesis may be dismissed without further consideration. For if plate thickness remains constant with age, the average plate density will increase with age, and this will also affect the height to which a volcano may grow according to isostasy. Vogt shows, however, that the height-age relationship expected in this case is quite unlike that actually observed.

\section{Pulsing X-ray stars}

from James Pringle

OF the sixty or so X-ray stars known in our Galaxy and in the Magellanic Clouds only a few have'so far been found to pulse regularly. One, the Crab pulsar, has a pulse period of 33 milliseconds, as do its radio and optical counterparts, and is thought to be a rapidly spinning neutron star. The star's large energy output, several thousand times that of the Sun, derives from its rotational kinetic energy; the pulse period is observed to increase on a time scale of about a thousand years.
Another two, Centaurus $X-3$ and Hercules $X-1$, pulse with periods of 4.84 and 1.24 seconds respectively, but, although their $\mathrm{X}$-ray luminosities are similar to that of the Crab pulsar, their pulse periods vary somewhat irregularly with no great evidence for overall speeding up or slowing down. Both these sources of $X$ rays are identified with optical stars. It is almost certain that the $X$ rays originate from a compact oompanion star, too faint to be visible optically, and that mass transfer from the 'normal' star on to its companion provides the source of energy. One strong possibility is that the compact star is a neutron star with a strong magnetic field (about 10 $0^{12}$ Gauss). Material falling on to such an object glides along the field lines on to the magnetic poles where it yields about $10 \%$ of its rest mass energy on striking the stellar surface. The necessary quantity of energy released in such a small area must lead to high enough temperatures to provide $\mathrm{X}$-ray emission. Rotation of the star, with its two X-ray hot spots, provides the pulse. Radiation produced in such a strong magnetic field should be highly polarised-an important observational test of this hypothesis.

An alternative possibility is that the compact object is a pulsating white dwarf star. For a neutron star, the gravitational energy released by the infall greatly exceeds the nuclear energy released by subsequent hydrogen burning. For a white dwarf of a solar mass, hydrogen burning releases 20 times as much energy as the infall, and even more for less massive stars. It has been suggested that this hydrogen burning could feed energy into pulsations of the star; these would then dissipate through shock waves in the stellar atmosphere. Preliminary work suggested that it was indeed possible to heat the white dwarf's atmosphere sufficiently to provide $\mathrm{X}$ rays of sufficient energy and intensity. But in a recent, and more detailed, calculation, Katz and Salpeter (Astrophysical Journal, 193, 429-436; 1974) conclude that these previous results were too optimistic and that any realistic calculation will give $\mathrm{X}$-ray emission too low by many orders of magnitude.

The authors consider radial pulsations of the star which, at a specified layer below the photosphere, are assumed to be sinusoidal in velocity. They stress that the peak downward acceleration of such a motion cannot be taken to be greater than the effective surface gravity: this condition has been violated in previous work. Also, because in general the scale height in the atmosphere is much less than the radius of the star, there is an 'acoustic mismatch' (and thus a very weak coupling) between the low stellar eigen- modes and atmospheric sound waves or shock waves. This mismatch can only be avoided if the luminosity of the star is close to the Eddington limit, in which case radiation pressure almost balances gravity and the atmospheric scale height is comparable with the stellar radius. In this event, however, the inverse Compton cooling (the sharing of energy between photons and electrons in the stellar atmosphere) produced by the stellar luminosity becomes most severe, and the shock heating of the atmosphere is suppressed. Compton cooling has been ignored in previous work and the present authors find it to be very important in damping atmospheric shock waves and removing energy from any shock heated material. These effects strongly inhibit the conversion of stellar oscillatory motion into multi-keV $\mathrm{X}$ rays.

The relevance of the above constraints to the non-pulsing $X$-ray sources is as yet unclear, although the authors do indicate some misgivings about the white dwarf hypothesis as a whole. On the other hand, there has been a tendency among theorists to ignore the possibility of X-ray emission from white dwarfs and it is likely that further progress will be made in this field before very long.

\section{Giant multipole resonances}

\section{from $P$. E. Hodgson}

MANY years ago it was found that the cross sections for the radiative capture of protons by nuclei showed broad maxima at energies around 10 to $20 \mathrm{MeV}$. These persist throughout the periodic table, and accurate measurements showed that the energies of these states is given approximately by $63 A^{-1 / 3} \mathrm{MeV}$.

Goldhaber and Teller (Phys. Rev., 74, 1046; 1948) explained these resonances as due to collective oscillations of the neutrons against the protons. This accounted both for their universal character and qualitatively for the variation of their energies from nucleus to nucleus. Subsequently a more detailed microscopic theory interpreted them as a quantum-mechanical superposition of particle-hole excitations giving a resonance of an El dipole character (Brown and Bolsterli, Phys. Rev. Lett., 3, 472; 1959; Elliott and Flowers, Proc. R. Soc., 242, $57 ; 1957)$.

Subsequently these dipole resonances were found to be excited in many other reactions, particularly by the inelastic scattering of electrons and protons (Nature, 246, 250; 1973), and detailed analyses of these data allowed many of the properties of the resonances to be determined. 